\title{
Contour integral method for European options with jumps
}

\author{
Edgard Ngounda, Kailash C. Patidar and Edson Pindza
}

\begin{abstract}
We develop an efficient method for pricing European options with jump on a single asset. Our approach is based on the combination of two powerful numerical methods, the spectral domain decomposition method and the Laplace transform method. The domain decomposition method divides the original domain into subdomains where the solution is approximated by using piecewise high order rational interpolants on a Chebyshev grid points. This set of points are suitable for the approximation of the convolution integral using Gauss-Legendre quadrature method. The resulting discrete problem is solved by the numerical inverse Laplace transform using the Bromwich contour integral approach. Through rigorous error analysis, we determine the optimal contour on which the integral is evaluated. The numerical results obtained are compared with those obtained from conventional methods such as Crank-Nicholson and finite difference. The new approach exhibits spectrally accurate results for the evaluation of options and associated Greeks. The proposed method is very efficient in the sense that we can achieve higher order accuracy on a coarse grid, whereas traditional methods would required significantly more time-steps and large number of grid points.
\end{abstract}

\section{Introduction}

In the general framework of the Black-Scholes model, the underlying stock price asset follows a geometric Brownian motion process and has a continuous sample path defined by

$$
\frac{d S}{S}=\mu d t+\sigma d W_{t}
$$

Here $S$ represents the underlying stock price at time $t$. It is assumed that the associated sample path is continuous. The constants $\boldsymbol{\mu}$ and $\sigma$ represent the expected return on the stock and the volatility of the return respectively; $d W t$ is the standard Brownian motion or a Wiener process. The Black-Scholes model predicts that the stock price $S$ follows a log-normal distribution at any future time $t$, i.e., 


$$
S(t)=S_{0} e^{\left(\left(\mu-\frac{\sigma^{2}}{2}\right) t+\sigma W_{t}\right)} .
$$

The continuity of the sample path indicates that the stock price can only change by a small amount in short interval. However, the reality on the stock market is different. Jumps are regularly observed in the discrete movement of the stock price St. These jumps cannot be capture by the log-normal distribution characteristic of the stock price in the Black-Scholes setting and therefore an alternative model which addresses this shortcoming is necessary.

To overcome the above mentioned shortcoming, a number of models have been proposed in the literature that more appropriately describe the movement of the stock price in the market. Among these, the jump-diffusion model proposed in [9] by Merton is one of the most widely used model. In this framework, the Brownian motion observed in the Black-Scholes model is combined with a poisson distribution which model the jumps discontinuities that normally occur on the market place. For the jumpdiffusion model, the movement of the stock price is therefore modeled by the following stochastic differential equation (SDE)

$$
\frac{d S}{S}=(\mu-\lambda \kappa) d t+\sigma d W_{t}+d q .
$$

As in the previous model, $\sigma$ represents the volatility, $\mu$ is the instantaneous expected return on the stock, and $\lambda$ is the intensity of the poisson precess (or the jump arrival rate), $d W t$ is the increment of the Brownian motion process, $\boldsymbol{K}=E \eta-1$ ), where $E$ is the expectation and $\mathrm{g}-1$ is the impulse producing the jump from $S$ to $S \eta$ if a Poisson event occurs and $d q$ is the independent Poisson process defined by

$$
d q=\left\{\begin{array}{lll}
0 & \text { with probability } 1-\lambda d t \\
1 & \text { with probability } & \lambda d t
\end{array}\right.
$$

Using the Itô formula, the SDE (1.2) is rewritten in the form of the following partial integrodifferntial equation (PIDE):

$$
\frac{\partial V}{\partial t}=\frac{1}{2} \sigma^{2} S^{2} \frac{\partial^{2} V}{\partial S^{2}}+(r-\lambda \kappa) S \frac{\partial V}{\partial S}-(r+\lambda) V+\lambda \int_{0}^{\infty} V(S \eta) \psi(\eta) d \eta .
$$

In the above, $\mathrm{V}(\mathrm{S} ; \mathrm{t})$ is the value of the option depending on the underlying stock price $\mathrm{S}$ at any given time $t$; $T$ is the expiry date, $r$ is the risk free interest rate $(r \geq 0), \lambda$ is the intensity of the Poisson process $(\lambda>0), j$ is the expected jump size, $t$ is the current time, $\Psi(\eta)$ is the probability function of the jump amplitude $\eta$, where $\Psi(\eta) \geq 0$, for all $\eta$, and is defined by 


$$
\psi(\eta)=\frac{e^{-\frac{(\log \eta-\mu)^{2}}{2 \gamma^{2}}}}{\sqrt{2 \pi} \gamma \eta} .
$$

Note that $\int_{0}^{\infty} \psi(\eta) d \eta=$ and when $\lambda=0$ in (1.3), we recover the standard Black-Scholes partial differential differential equation.

For European options, Merton [9] derived analytical expressions but for most exotic options under jump-diffusion models, no closed-form solutions exist and one needs to find numerical solutions for the partial integro-differential equations that arise. However, the convolution integral (1.3) add to the difficulty of finding efficient numerical solutions. Commonly used finite difference methods (FDMs) hardly attain higher order accuracy [2] and typical quadrature rules such as the trapezoidal and Simpson's rules are of low order compared to Gaussian quadrature. However, the later is expensive to implement since it requires the interpolation to match the Chebyshev grid point with those of the FDMs. To reduce the computational cost in solving the convolution integral term, Fast Fourier Transform (FFT) was used in [2,19].

Tangman et al. [14] proposed a different approach in combining the central difference method and the exponential time differencing (ETD) scheme to solve (1.3). The ETD method was proved as very effective and gave second order accuracy. This successful result, encouraged these authors to apply higher order discrete method such as spectral methods to enhance the spatial convergence of the solution. To get around the nonsmooth initial condition, a cluster grid of Chebyshev points at the discontinuous point and at boundaries were performed and they obtained fourth order results.

Spectral method are attractive for their exponential convergence rate. This presents an advantage for a direct computation of the convolution integral by a high order Gauss quadrature method. However, high rate of convergence of the spectral method is only guaranteed for smooth solution, a condition which is not fulfilled for the jump-diffusion model (1.3) which has a non-smooth initial condition.

To overcome this situation, one might consider using a spectral element approach. This is the approach followed in [18] where the PIDE (1.3) is solved and the resulting discrete ODE is integrated in time using Crank-Nicolson method. This resulted in spectrally accurate results in space and second order accuracy in time. The exponential results are partly due to the successful approximation of the integral term by Gauss quadrature rule. However, the application of the spectral element involved successive approximation of different integrals generated by the weak form and hence computationally expensive.

In this paper, we propose the use of a multi-domain spectral method. This method uses the spectral method directly in each sub-domains. Matching conditions are imposed to ensure the continuity of the solution and that of its first derivative. After this spatial 
discretization, the resulting system of ODEs is solved by the Laplace transformation. To recover the solution, an inversion of the Laplace transform solution is performed using the Talbot's method [13] which is based on the application of trapezoidal rule to approximate a Bromwich integral.

The rest of this paper is organized as follows. In Section 2, we give a description of the jump-diffusion model and derive formula for the resolution of the convolution integral. In Section 3, we describe the spectral domain decomposition method for the differential part as well as the integral part. The later is computed by the Gauss-Legendre quadrature. Section 4 deals with the application of the Laplace transform to solve the semi-discrete problem. We also discuss the error analysis related to this approximation in this section. Section 5 contains the numerical comparisons of results obtained by approach with more conventional methods such as Crank-Nicholson for time integration and finite difference for space discretization. Some concluding remarks and scope for future research are given in Section 6.

\section{Description of model problem}

We consider the PIDE (1.3) and apply the change of the variable $s=\log (\mathrm{S} / \mathrm{K})$ and $\eta=\mathrm{e}^{\mathrm{y}}$. This gives

$$
\frac{\partial V}{\partial t}=\frac{1}{2} \sigma^{2} \frac{\partial^{2} V}{\partial s^{2}}+\left(r-\frac{\sigma^{2}}{2}-\lambda \kappa\right) \frac{\partial V}{\partial s}-(r+\lambda) V+\lambda \int_{-\infty}^{\infty} V(s+y) \widetilde{\psi}(y) d y .
$$

In the above, $V(s+y) \equiv V(t, s+y)$, i.e., $V$ is a function of $t$ and variables $s$ and $y$, with $s$ $E(-\infty ; \infty)$ and $\sigma>0$ is the volatility, $r \geq 0$ is the risk-free interest rate, $\lambda>0$ is the intensity of the Poisson process (or the jump). The jump probability density function is

$$
\widetilde{\psi}(y)=\frac{e^{\frac{(y-\mu)^{2}}{2 \gamma^{2}}}}{\sqrt{2 \pi} \gamma},
$$

while the expected jump size $k$ is

$$
\kappa=e^{\mu+\frac{\gamma^{2}}{2}}-1
$$

Let $\Omega=$ [smin; Smax] be the truncated interval and $\Omega^{\mathrm{c}}=\mathrm{R} \backslash$ [smin; smax $]$ its complement in R. To facilitate the computation of the integral part, we note that 


$$
\begin{aligned}
\int_{-\infty}^{\infty} V(s+y) \widetilde{\psi}(y) d y & =\int_{-\infty}^{\infty} V(y) \widetilde{\psi}(y-s) d y \\
& =\int_{-s_{\min }}^{s_{\max }} V(y) \widetilde{\psi}(y-s) d y+\int_{\Omega^{c}} V(y) \widetilde{\psi}(y-s) d y
\end{aligned}
$$

Where

$$
\int_{\Omega^{c}} V(y) \widetilde{\psi}(y-s) d y=\int_{-\infty}^{s_{\min }} V(y) \widetilde{\psi}(y-s) d y+\int_{s_{\max }}^{\infty} V(y) \widetilde{\psi}(y-s) d y
$$

For a European style put option, the boundary condition

$$
V(0, t)=K e^{-r t} \quad \text { is changed to } V(s, t)=K e^{-r t}, \quad s \rightarrow-\infty,
$$

And

$$
V(S, t)=0, \quad S \rightarrow \infty \text { becomes } \quad V(s, t)=0, \quad s \rightarrow \infty .
$$

Using these boundary conditions, and setting $w=(y-s-\mu) / \sigma$ so that $d w=d y / \sigma$, we obtain the following outer integral from (2.2):

$$
\int_{\Omega^{c}} V(y) \widetilde{\psi}(y-s) d y=\int_{-\infty}^{s_{\min }} V(y) \widetilde{\psi}(y-s) d y=K e^{-r t} \int_{-\infty}^{s_{\min }} \frac{e^{\frac{y-s-\mu^{\prime}}{2 \sigma^{2}}}}{\sigma \sqrt{2 \pi}} d y=K e^{-r t} \int_{-\infty}^{s_{\min }-s-\mu} \frac{e^{-w^{2}}}{\sqrt{2 \pi}} d w=K e^{-r t} \Phi\left(\frac{s_{\min }-s-\mu}{\sigma}\right),
$$

where $\Phi($.$) is the normal cumulative distribution function.$

For a European style call option, we observe that boundary condition

$$
V(0, t)=0 \quad \text { is changed to } V(s, t)=0, \quad s \rightarrow-\infty,
$$

And

$$
V(S, t) \rightarrow S-K e^{-r t}, \quad S \rightarrow \infty \text { becomes } \quad V(s, t) \rightarrow K e^{s}-K e^{-r t}, \quad s \rightarrow \infty .
$$

Again setting $w=(y-s-\mu) / \sigma$ and $d w=d y=\sigma$, the outer integral in (2.2) leads to 


$$
\begin{aligned}
\int_{\Omega^{c}} V(y) \widetilde{\psi}(y-s) d y & =K \int_{s_{\max }}^{\infty} e^{y} \widetilde{\psi}(y-s) d y-K e^{-r t} \int_{s_{\max }}^{\infty} \widetilde{\psi}(y-s) d y=K \int_{s_{\max }}^{\infty} e^{y} \frac{e^{-\frac{(y-s-\mu)^{2}}{2 \sigma^{2}}}}{\sigma \sqrt{2 \pi}} d y-K e^{-r t} \int_{s_{\max }}^{\infty} \frac{e^{-\frac{(y-s-\mu)^{2}}{2 \sigma^{2}}}}{\sigma \sqrt{2 \pi}} d y \\
& =K \frac{e^{s+\mu}}{\sqrt{2 \pi}} \int_{\frac{s_{\max }-s-\mu}{\sigma}}^{\infty} e^{-\frac{1}{2}(w-\sigma)^{2}+\frac{\sigma^{2}}{2}} d w-\frac{K e^{-r t}}{\sqrt{2 \pi}} \int_{\frac{s_{\max }-s-\mu}{\sigma}}^{\infty} e^{-\frac{w^{2}}{2}} d w \\
& =K \frac{e^{s+\mu+\frac{\sigma^{2}}{2}}}{\sqrt{2 \pi}} \int_{\frac{s_{\max }-s-\mu}{\sigma}-\sigma}^{\infty} e^{-\frac{w^{2}}{2}} d w-\frac{K e^{-r t}}{\sqrt{2 \pi}} \int_{\frac{s_{\max }-s-\mu}{\sigma}}^{\infty} e^{-\frac{w^{2}}{2}} d w \\
& =K \frac{e^{s+\mu+\frac{\sigma^{2}}{2}}}{\sqrt{2 \pi}} \int_{-\infty}^{\frac{s-s_{\max }+\mu+\sigma^{2}}{\sigma}} e^{-\frac{w^{2}}{2}} d w-\frac{K e^{-r t}}{\sqrt{2 \pi}} \int_{-\infty}^{\frac{s-s_{\max }+\mu}{\sigma}} e^{-\frac{w^{2}}{2}} d w \\
& =K e^{s+\mu+\frac{\sigma^{2}}{2}} \Phi\left(\frac{s-s_{\max }+\mu+\sigma^{2}}{\sigma}\right)-K e^{-r t} \Phi\left(\frac{s-s_{\max }+\mu}{\sigma}\right) .
\end{aligned}
$$

In the next section, we use the Spectral domain decomposition method (SDDM) to discretize the jump-diffusion model (1.3).

\section{Spectral domain decomposition method}

The main feature of spectral methods is that approximation errors associated with the methods often decay exponentially with increasing numbers of degrees of freedom. In contrast, errors associated with finite difference methods and classical finite element methods decay only algebraically. If the error decays exponentially, then a result that is accurate to, say, 10 digits can be obtained using fewer degrees of freedom than would have been required if the error had decayed algebraically. This suggests that spectral methods are often more efficient than finite difference and classical finite element methods. However, the exponential convergence rate of spectral method is strictly dependent on the smoothness of the unknown function. Option pricing problems involve PDEs with initial conditions that are non-smooth. Therefore, direct application of a spectral method to the solution of such equations usually lead to a low order approximation.

To overcome this, we propose the spectral domain decomposition method (SDDM). In the spectral domain decomposition approach, the domain s $E$ [smin; smax] is divided into sub-domains. For the problem at hand, we need to divide the domain in two subdomains, with the transition point as the strike price. To represent the solution in each sub-domain, we choose to approximate the solution by a linear rational interpolants for their improved stability properties compared to the polynomial interpolants [3,15].

\subsection{Spectral domain decomposition based on rational interpolants}

Domain decomposition is a discretization technique for solving differential equations whereby the computational domain is divided into a number of smaller sub-domains. The equation is then solved on each sub-domain with matching conditions enforced at the interface (or transition point $\mathrm{K}$ ) to ensure the continuity of the solution and that of it first derivative across the sub-domains. In the context of spectral methods on an interval, this means that rather than using a single global polynomial on the entire domain, polynomials of different order may be used on each subinterval. However in this work, rather than using polynomial interpolants for spectral methods, we will

\section{http://repository.uwc.ac.za}


consider the more stable linear rational interpolants. For further discussion on these types of interpolants, readers are referred [3,15].

\subsubsection{Rational interpolants}

Rational interpolants are more convenient for the problem at hands. The rational approximation of a function $u(\xi)$ at the Chebyshev points $\xi$, for $k=0 ; 1 ; \ldots ; N$, is given by

$$
u_{N}(\xi)=\frac{\sum_{k=0}^{N} \frac{w_{k}}{\xi-\xi_{k}} u\left(\xi_{k}\right)}{\sum_{k=0}^{N} \frac{w_{k}}{\xi-\xi_{k}}}
$$

where wk, for $\mathrm{k}=0 ; 1 ; \ldots ; \mathrm{N}$, are the barycentric weights defined as wo $=1 / 2 ; \mathrm{wN}=(-1)^{\mathrm{N}} / 2$, and $\mathrm{wk}=(-1)^{\mathrm{K}}, \mathrm{k}=1 ; \ldots ; \mathrm{N}-1$. The Chebyshev points are define as

$$
\xi_{k}=\cos \left(\frac{k \pi}{N}\right), \quad k=0,1, \ldots, N
$$

As for the spectral polynomial method, the m-th order differentiation matrix associated with the rational interpolant (3.1) is given by

$$
u_{N}^{(m)}\left(\xi_{k}\right)=\sum_{k=0}^{N} \frac{d^{m}}{d \xi^{m}}\left(\frac{\sum_{k=0}^{N} \frac{w_{k}}{\xi_{k} \xi_{k}} u\left(\xi_{k}\right)}{\sum_{k=0}^{N} \frac{w_{k}}{\xi-\xi k}}\right)=\sum_{k=0}^{N} D_{j k}^{(m)} u\left(\xi_{k}\right),
$$

Where $D_{j k}^{(m)}$ are the entries of the differentiation matrix of order m. Formula to construct $D_{N}^{(m)}$ were given by Schneider and Werner [11] for $\mathrm{m}=1$ and $\mathrm{m}=2$ and latter generalized for any order by Tee in [15]. The first and second order differentiation matrices are given by the following formulas

$$
D_{j k}^{(1)}= \begin{cases}\frac{w_{k}}{w_{j}\left(\xi_{j}-\xi_{k}\right)}, & j \neq k, \\ -\sum_{i \neq k} D_{j i}^{(1)}, & j=k,\end{cases}
$$

and

$$
D_{j k}^{(2)}= \begin{cases}2 D_{j k}^{(1)}\left(D_{j j}^{(1)}-\frac{1}{\xi_{j}-\varepsilon_{k}}\right), & j \neq k, \\ -\sum_{i \neq k} D_{j i}^{(2)}, & j=k .\end{cases}
$$

We apply the rational interpolant along with the domain decomposition method (as described below) to solve the jump-diffusion call option (2.1), (2.5) and (2.6). 


\subsubsection{Discretization of the jump-diffusion model}

We split the domain into two sub-domains at the point of discontinuity, and apply Chebyshev spatial discretization to reduce the problem to a set of coupled ordinary differential equations in time. Since the function is smooth on each subinterval including the point of discontinuity, a spectral accuracy can be regained provided that appropriate matching conditions are set across the point of discontinuity.

Since the initial condition is non-smooth at the strike price, we split the interval [smin; smax] at o into two subintervals: $v=[\operatorname{smin}, \mathrm{o}]$ and $\mathrm{x}=\left[\mathrm{o}, \mathrm{S}_{\max }\right]$ of lengths $d^{v}=$ smin and $d^{\mathrm{X}} s_{\max }$, respectively. To apply the Chebyshev discretization, we map each sub-domain to the reference element $[-1 ; 1]$ by the linear transformation

$$
s^{\vartheta}, \mathcal{\chi}(\xi)= \begin{cases}\frac{d^{\natural}}{2}(1-\xi), & s \in\left[s_{\min }, 0\right], \\ \frac{d^{\chi}}{2}(\xi+1), & s \in\left[0, s_{\max }\right] .\end{cases}
$$

The linear transformation to its derivative leads to

$$
d s^{\vartheta, \chi}(\xi)=\left\{\begin{array}{l}
-\frac{d^{\natural}}{2} d \xi, \quad s \in\left[s_{\min }, 0\right], \\
\frac{d^{\chi} \chi}{2} d \xi, \quad s \in\left[0, s_{\max }\right] .
\end{array}\right.
$$

Thus

$$
\frac{\partial}{\partial s} \equiv \frac{\partial}{\partial \xi} \frac{\partial \xi}{\partial s} \equiv \begin{cases}-\frac{2}{d^{j}} \frac{\partial}{\partial \xi}, & s \in\left[s_{\min }, 0\right] \\ \frac{2}{d^{x}} \frac{\partial}{\partial \xi}, & s \in\left[0, s_{\max }\right] .\end{cases}
$$

In the following, we use the spectral domain decomposition method to the model problem (2.1) subject to the boundaries (2.5) and (2.6). To keep the expressions simple, we first discretize the differential part of (2.1) and thereafter the integral part.

On each sub-domains $\vartheta$ and $\mathrm{x}$, the differential part of (1.3) is discretized as

$$
\begin{aligned}
& \text { and } \begin{aligned}
\frac{\partial V^{\vartheta}}{\partial \tau} & =\frac{2}{\left(d^{\vartheta}\right)^{2}} \sigma^{2} \frac{\partial^{2} V^{\vartheta}}{\partial \xi^{2}}-\frac{2}{d^{\vartheta}}\left(r-\lambda \kappa-\frac{\sigma^{2}}{2}\right) \frac{\partial V^{\vartheta}}{\partial \xi}-(r+\lambda) V^{\vartheta}, \\
\frac{\partial V^{\vartheta}}{\partial \tau} & =\frac{2}{\left(d^{\vartheta}\right)^{2}} \sigma^{2} \frac{\partial^{2} V^{\vartheta}}{\partial \xi^{2}}-\frac{2}{d^{\vartheta}}\left(r-\lambda \kappa-\frac{\sigma^{2}}{2}\right) \frac{\partial V^{\vartheta}}{\partial \xi}-(r+\lambda) V^{\vartheta} .
\end{aligned}
\end{aligned}
$$

In matrix form we have

$$
\dot{V}^{\vartheta}=\frac{2}{\left(d^{\vartheta}\right)^{2}} \sigma^{2} D^{(2)} V^{\vartheta}-\frac{2}{d^{\vartheta}}\left(r-\lambda \kappa-\frac{\sigma^{2}}{2}\right) D^{(1)} V^{\vartheta}-(r+\lambda) V^{\vartheta},
$$

and

$$
\dot{V}^{\chi}=\frac{2}{\left(d^{\chi}\right)^{2}} \sigma^{2} D^{(2)} V^{\chi}-\frac{2}{d^{\chi}}\left(r-\lambda \kappa-\frac{\sigma^{2}}{2}\right) D^{(2)} V^{\chi}-(r+\lambda) V^{\chi}
$$

Here $\dot{V}^{\vartheta, \chi}$ is the derivative of $\mathrm{V}$ with respect to $\mathrm{s}$ in the sub-domains $\vartheta$ and $\mathrm{x}$ respectively. Note that the derivatives of the same order from both sub-domains would be different if rational interpolants of different order are chosen in each sub-domain. 
The above equations imply

$$
\dot{V}^{\theta, \mathcal{X}}=B^{\theta, \chi} V^{\theta, \chi},
$$

where

$$
B^{\theta, \chi}=\frac{2}{\left(d^{0, \mathcal{X}}\right)^{2}} \sigma^{2} D^{(2)}-\frac{2}{d^{0, \mathcal{X}}}\left(r-\lambda \kappa-\frac{\sigma^{2}}{2}\right) D^{(1)}-(r+\lambda) I^{\beta, \chi} .
$$

and $I^{\vartheta, \chi}$ is the $(N+1) \mathrm{x}(N+1)$ identity matrix.

Now we consider the approximation of the integral part in (1.3). We know that for a call option the indefinite integral gives

$$
\int_{-\infty}^{\infty} V(s+y) \widetilde{\psi}(y) d y=\int_{s_{\min }}^{s_{\max }} V(y) \widetilde{\psi}(y-s) d y+K e^{s+\mu+\frac{\sigma^{2}}{2}} \Phi\left(\frac{s-s_{\max }+\mu+\sigma^{2}}{\sigma}\right)-K e^{-r t} \Phi\left(\frac{s-s_{\max }+\mu}{\sigma}\right) .
$$

Hence we need only to discretize the integral on the right of the equation. For simplicity, we consider the same number of grid points in each sub-domains $\vartheta$ and $\mathrm{x}$. This gives

$$
\int_{s_{\min }}^{s_{\max }} V(z) \tilde{\psi}(z-s) d z=\int_{s_{\min }}^{0} V\left(z^{\vartheta}\right) \widetilde{\psi}^{\vartheta}\left(z^{\vartheta}-s\right) d z+\int_{0}^{s_{\max }} V\left(z^{\chi}\right) \widetilde{\psi}^{\chi}\left(z^{\chi}-s\right) d z=H^{\vartheta}+H^{\chi}
$$

Mapping the two intervals $[\operatorname{Smin}, 0]$ and $[0, \operatorname{Smax}]$ to the standard element $[-1 ; 1]$ by the linear transformation

$$
z^{\ominus * \chi}(\xi)= \begin{cases}\frac{d^{\natural}}{2}(1-\xi), & z^{0} \in\left[s_{\min }, 0\right], \\ \frac{d^{\chi}}{2}(\xi+1), & z^{\chi} \in\left[0, s_{\max }\right]\end{cases}
$$

we obtain

$$
H^{\vartheta}=-\frac{s_{\min }}{2} \int_{-1}^{1} V\left(z^{\vartheta}\right) \tilde{\psi}^{\vartheta}\left(z^{\vartheta}-s\right) d z^{\vartheta}
$$

and

$$
H^{*}=\frac{s_{\max }}{2} \int_{-1}^{1} V\left(z^{\chi}\right) \tilde{\psi}^{\chi}\left(z^{\chi}-s\right) d z^{\chi}
$$

Finally, since the grid points are Chebyshev, a well suited approximation method to evaluate integrals $H^{\vartheta}$ and $H^{\chi}$ is the Gauss-Legendre quadrature rule, which converges geometrically [16]. Making use of this rule in each sub-domain, the two terms on the right hand side of (3.13) given by (3.15) and (3.16) are approximated as 


$$
\begin{aligned}
& H^{\vartheta}=-\frac{s_{\min }}{2} \sum_{k=0}^{N} V\left(z^{\vartheta}\right) \tilde{\psi}^{\vartheta}\left(z^{\vartheta}-s\right) \varpi_{k}, \\
& H^{\chi}=\frac{s_{\max }}{2} \sum_{k=0}^{N} V\left(z^{\chi}\right) \tilde{\psi}^{\chi}\left(z^{\chi}-s\right) \varpi_{k},
\end{aligned}
$$

Where $\varpi_{k}$,for $\mathrm{k}=0 ; 1 ; \ldots ; \mathrm{N}$ denotes the Gauss-Legendre quadrature weights.

On the sub-domain $\vartheta$, the discretization of the differential and integral parts yields

$$
\dot{V}^{\vartheta}=\frac{2}{\left(d^{\vartheta}\right)^{2}} \sigma^{2} D^{(2)} V^{\vartheta}+\frac{2}{d^{\vartheta}}\left(r-\lambda \kappa-\frac{\sigma^{2}}{2}\right) D^{(1)} V^{\vartheta}-(r+\lambda) V^{\vartheta}-\frac{s_{\min }}{2} \sum_{k=0}^{N} V\left(z^{\vartheta}\right) \widetilde{\psi}^{\vartheta}\left(z^{\vartheta}-s\right) \varpi_{k},
$$

whereas on the sub-domain $\chi$ we have

$$
\dot{V}^{\chi}=\frac{2}{\left(d^{\chi}\right)^{2}} \sigma^{2} D^{(2)} V^{\chi}-\frac{2}{d^{\chi}}\left(r-\lambda \kappa-\frac{\sigma^{2}}{2}\right) D^{(1)} V^{\chi}-(r+\lambda) V^{\chi}+\frac{s_{\max }}{2} \sum_{k=0}^{N} V\left(z^{\chi}\right) \widetilde{\psi}^{\chi}\left(z^{\chi}-s\right) \varpi_{k} .
$$

Upon simplification, (3.19) and (3.20) give

$$
\dot{V}^{\vartheta}=B^{\vartheta} V^{\vartheta}+\lambda H^{\vartheta} V^{\vartheta}, \quad \text { and } \quad \dot{V}^{\chi}=B^{\chi} V^{\chi}+\lambda H^{\chi} V^{\chi} \text {. }
$$

To impose the boundary conditions, we substitute the first and last equations from the first and second sub-domains, respectively, by the boundary conditions (2.3) and (2.4). Furthermore, to ensure the continuity of the solution and that of its first derivatives at the interface, we impose matching conditions across the point of discontinuity, i.e., at $\mathrm{s}$ $=\mathrm{O}$,

$$
V^{*}=V_{N}^{\vartheta}=V_{0}^{\chi}, \quad \text { and } \quad \frac{\partial V_{N}^{\vartheta}}{\partial \xi}=\frac{\partial V_{0}^{\chi}}{\partial \xi} .
$$

The first matching condition leads to

$$
2 \dot{V}^{*}=\left[\left(B^{\vartheta}+\lambda H^{\vartheta}\right) V^{\vartheta}\right]_{N}+\left[\left(B^{\chi}+\lambda H^{\chi}\right) V^{\chi}\right]_{0} .
$$

From the second condition above we have

$$
\frac{2}{d^{\vartheta}}\left(D^{(1)} V^{\vartheta}\right)_{N}-\frac{2}{d^{\chi}}\left(D^{(1)} V^{\chi}\right)_{0}=0 .
$$

Finally, the discrete approximation of the jump-diffusion model (2.1) is obtained by combining the approximations from the two sub-domains with boundary and matching conditions included into a global system. This gives

$$
\dot{V}=(B+\lambda H) V+\epsilon_{1}(s)-\epsilon_{2}(s) e^{-r \tau},
$$

where $V$ is the vector

$$
V=\left[V_{0}^{\vartheta}, \ldots, V_{N}^{\vartheta}, V_{0}^{\chi}, \ldots, V_{N}^{\chi}\right],
$$

$B, H$ are block matrices of $B^{\vartheta, \chi}$ and $H^{\vartheta, \chi}$ respectively; and

$$
\epsilon_{1}(s)=\lambda K e^{s+\mu+\frac{\sigma^{2}}{2}} \Phi\left(\frac{s-s_{\max }+\mu+\sigma^{2}}{\sigma}\right),
$$

And 


$$
\epsilon_{2}(s)=\lambda K e^{-r t} \Phi\left(\frac{s-s_{\max }+\mu}{\sigma}\right)
$$

After this discretization, the resulting system of ODEs is solved by using the Laplace transform approach which we discuss in the next section.

\section{Application of Laplace transform to solve the semi-discrete problem}

The Laplace transform is a powerful tool for solving PDEs. However the main difficulty associated with this method is the reconstruction of the original function $f(t)$ for a given Laplace transform $\mathrm{F}(\mathrm{s})$. Unless the inverse is given explicitly, one has to evaluate the Bromwich integral:

$$
f(t)=\frac{1}{2 \pi i} \int_{\Gamma} e^{2 t} F(z) d z, \quad t>0 .
$$

The contour $\Gamma$ is known as the Bromwich contour. Originally $\Gamma$ represents the vertical line from $\tilde{\sigma}_{0}-i \infty$ to $\tilde{\sigma_{0}}+i \infty$ with $\tilde{\sigma}>\tilde{\sigma}_{0}$, where

$$
F(z)=\int_{0}^{\infty} e^{-z t} f(t) d t, \quad \operatorname{Re} z>\tilde{\sigma}_{0},
$$

and $\tilde{\sigma}_{0}$ the convergence abscissa of the Laplace transform with $\tilde{\sigma} \geq \tilde{\sigma}_{0}$. Formula (4.1) is valid if all singularities of $F(z)$ are located to the left of the vertical line $x=\tilde{\sigma}$, i.e., $\operatorname{Re} z<$ $\tilde{\sigma}$. This is the case for parabolic problems such as the PIDE we are solving.

We assume that $f(t)$ is a function of exponential order as $t \rightarrow+\infty, \tilde{\sigma}_{0}$ such that $e^{-\tilde{\sigma}_{0} t}|f(t)| \leqslant+\infty$. In addition, if $f(t)$ is absolutely integrable for $t>0$, then the Laplace integral (4.2) converges for all $\operatorname{Re} z>\tilde{\sigma}_{0}$. The main difficulty, however, in the use of the Laplace transform comes from the recovery of the original function $f(t)$, i.e., the evaluation of the inverse formula (4.1). Analytical solutions are often hard to implement, and one has to rely on numerical methods to evaluate (4.1). An inventory of some of the most significant numerical methods developed in the last three decades can be found in $[4,6,10]$. These methods were classified by Abate and Valko [1] as Fourier series expansion method, Laguerre function expansion method, method based on the combination of Gaver functionals, and method based on the deformation of the Bromwich contour. In this paper, we use the last of these four methods.

For the numerical evaluation of (4.1), we consider the parametrization

$$
z=\tilde{\sigma}+i y, \quad-\infty<y<\infty .
$$

The integral (4.1) can then be rewritten as

$$
f(t)=\frac{e^{\tilde{\sigma} t}}{2 \pi i} \int_{-\infty}^{\infty} e^{i y t} F(\tilde{\sigma}+i y) d y .
$$


Unfortunately, in most cases, the above integral is difficult to evaluate numerically for a number of reasons: firstly, the integrand is highly oscillatory on the Bromwich contour (4.3), when $y \rightarrow \pm \infty$. Secondly, the transformed function $F(\tilde{\sigma}+i y)$ may decay slowly as $y \rightarrow \pm \infty$ ([5]).

Our numerical method for inverting the Laplace transform is based on the method developed by Talbot [13] who proposed the deformation of the Bromwich contour. The integral is then evaluated using the trapezoidal rule. Talbot's idea was to deform the Bromwich line into a contour which starts and ends in the left half-plane (see Fig. 1 for an example of such a contour). Such a deformation of the contour is possible by Cauchy's integral theorem [12]. Cauchy's theorem is applicable provided that all singularities of the transformed function $F(z)$ are contained in the interior of the new contour and that $|F(z)| \rightarrow 0$ as $|z| \rightarrow \infty$ in the half-plane $\operatorname{Re} z<\tilde{\sigma}_{0}$ [17]. Such contours are used in $[7,13,17]$, all of which are of the form

$$
z=z(\ell), \quad-\infty<\ell<\infty
$$

with the property that $\operatorname{Rez} \rightarrow-\infty$ as $\ell \rightarrow \pm \infty$.

The efficiency of the Talbot approach depends on the choice of the contour, as well as the number of function evaluations in the trapezoidal rule. Simpler contours such as hyperbolas and parabolas are proposed in $[7,17]$. These contours display a better convergence rate of order $\mathcal{O}\left(10^{-N}\right)$ than the original cotangent contour from Talbot. In this paper, we consider the hyperbola as the integration contour defined by

$$
z=\tilde{\mu}(1+\sin (i \ell-\alpha)), \quad \ell \in \mathbb{R},
$$

where the real parameters $\mu>0$ and $0<\alpha<\pi / 2$ determine the geometry of the contour. The positive parameter $k$ controls the width of the contour while $\alpha$ determines its geometric shape, i.e., the asymptotic angle. On the contour (4.5) the inversion formula (4.1) can be rewritten as

$$
f(t)=\frac{1}{2 \pi i} \int_{-\infty}^{\infty} e^{z(\ell) t} F(z(\ell)) z^{\prime}(\ell) d \ell
$$




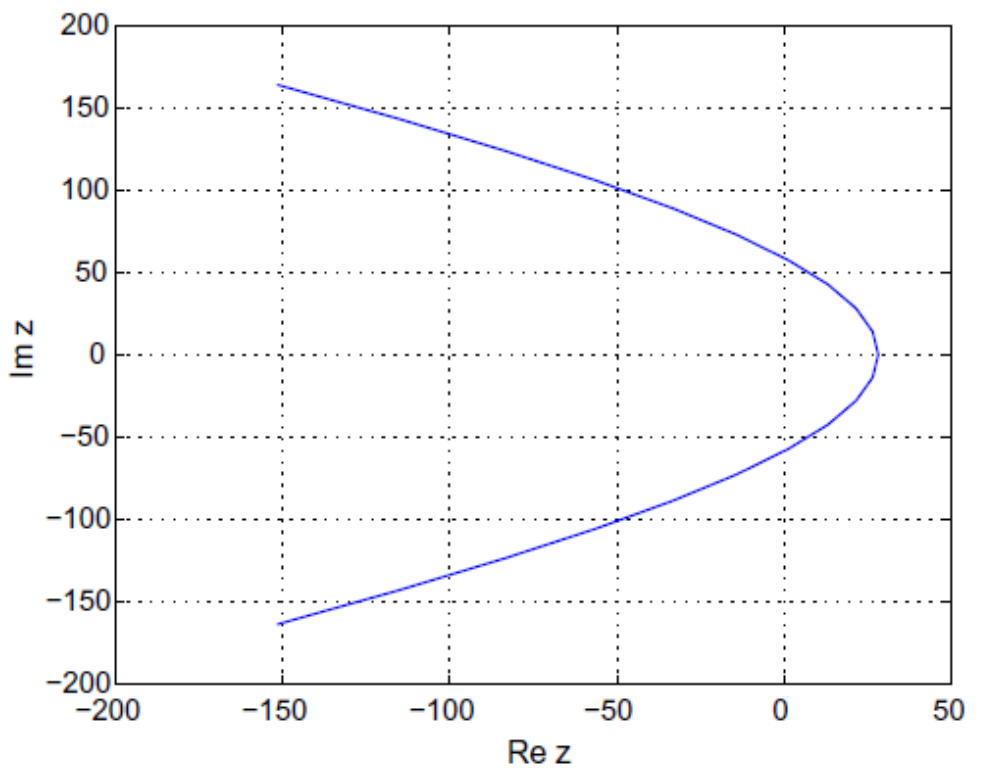

Fig. 1. Talbot contour of hyperbolic type.

Where

$$
z^{\prime}(\ell)=\tilde{\mu} i \cos (i \ell-\alpha)
$$

For $\mathrm{h}>\mathrm{o}$ such that $\ell_{k}=k h$, where $\mathrm{k}$ is an integer, the trapezoidal rule can then be expressed by

$$
\tilde{f}(t) \approx \frac{h}{2 \pi i} \sum_{k=-\infty}^{\infty} e^{2\left(\ell_{k}\right) t} F\left(z\left(\ell_{k}\right)\right) z^{\prime}\left(\ell_{k}\right)
$$

In practice, the infinite sum has to be truncated at a finite integer $M$, in which case one commits a truncation error as discussed below. Note that, because of the symmetry of the contour (4.5), we can re-write (4.7) as

$$
\tilde{f}_{M}(t) \approx \operatorname{Re}\left\{\frac{h}{\pi i} \sum_{k=0}^{M} e^{0} e^{z\left(\ell_{k}\right) t} F\left(z\left(\ell_{k}\right)\right) z^{\prime}\left(\ell_{k}\right)\right\}
$$

where ${ }^{0}$ indicates that the first term is divided by 2. The benefit of using (4.8) is that it reduces by half the summation (4.7) and subsequently the number of linear system to be evaluated in (4.35).

In the following subsections, we analyse the overall error that occurs during the approximation of the solution using our method of integration in time.

\section{http://repository.uwc.ac.za}




\subsection{Analysis of the error}

In this section, we analyze the error associated with the use of Laplace transform for integration in the time direction. To this end, first we note that the application of the trapezoidal rule (4.7) to approximate the infinite integral (4.6) introduces discretization error. Secondly the truncation of the infinite series (4.7) at a finite integer M (for practical implementation) produces a truncation error as one would expects. Furthermore, since the evaluation of (4.7) is done in floating point environment, a roundoff error is also introduced at each evaluation there. This roundoff error may increase dramatically to affect the accuracy of the numerical solution due to the exponential factor involved in (4.7). The analysis of the error occurred due to the use of the Laplace transform is therefore the sum of the discretization, truncation and conditioning errors as we discuss below.

\subsubsection{Discretization error}

The discretization error is the difference between the continuous formula (4.6) and the corresponding trapezoidal formula (4.7), i.e.,

$$
E_{d}=f(t)-\tilde{f}(t)=\int_{-\infty}^{\infty} e^{z(\ell) t} F(z(\ell)) z^{\prime}(\ell) d \ell-\frac{h}{2 \pi i} \sum_{k=-\infty}^{\infty} e^{z\left(\ell_{k}\right) t} F\left(z\left(\ell_{k}\right)\right) z^{\prime}\left(\ell_{k}\right)
$$

To estimate the discrete error (4.9), the idea is to use the contour integral to represent $E_{d}$. This approach based on the Cauchy's Residue theorem in complex analysis was originally developed in [8] by Martensen for an analytic function $f(t)$ defined on $(-\infty ; \infty)$. In that paper, the author showed that for an analytic function, the trapezoidal rule (4.7) converges exponentially as illustrated in the following theorem.

Theorem 4.1. Let $f: \mathbb{R} \rightarrow \mathbb{R}$ be an analytic function. Then there exists a strip $\mathbb{R} \times(-d$; d) in the complex plane with $d>0$ such that $f$ can be extended to a complex analytic function $f: \mathbb{R} \times(-d ; d) \rightarrow \mathbb{C}$. Furthermore, the error for the trapezoidal rule indicated 4.9), is given by

$$
E_{d}=\operatorname{Re}\left\{\int_{-\infty+i \theta}^{\infty+i \theta}\left(1-i \cot \frac{\pi z}{h}\right) f(z) d z\right\},
$$

where $0<\theta<d$. Moreover, it is bounded by

$$
\left|E_{d}\right| \leqslant \frac{2 C}{e^{2 \pi d / h}-1},
$$

where $C$ is a constant such that

$$
\int_{-\infty+i \theta}^{\infty+i \theta}|f(z)| d z \leqslant C .
$$

Proof (See [8]). From the above theorem, it is clear that 


$$
\left|E_{d}\right| \leqslant \frac{2 e^{-2 \pi d / h} C}{1-e^{-2 \pi d / h}} \rightarrow 0 \quad \text { as } \quad h \rightarrow 0 .
$$

\subsubsection{Truncation error}

The truncation error is the error made by ignoring the remaining terms in (4.7) after truncating the series at a finite number $M$, and is given by

$$
\begin{aligned}
E_{t} & =h \sum_{k=-\infty}^{\infty} e^{z\left(\ell_{k}\right) t} F\left(z\left(\ell_{k}\right)\right) z^{\prime}\left(\ell_{k}\right)-\frac{h}{2 \pi i} \sum_{k=-M}^{M} e^{z\left(\ell_{k}\right) t} F\left(z\left(\ell_{k}\right)\right) z^{\prime}\left(\ell_{k}\right) \\
& =\frac{h}{2 \pi i}\left(\sum_{k=-\infty}^{M-1} e^{z\left(\ell_{k}\right) t} F\left(z\left(\ell_{k}\right)\right) z^{\prime}\left(\ell_{k}\right)+\sum_{k=M+1}^{\infty} e^{z\left(\ell_{k}\right) t} F\left(z\left(\ell_{k}\right)\right) z^{\prime}\left(\ell_{k}\right)\right) .
\end{aligned}
$$

Note that we have $\overline{F\left(z\left(\ell_{k}\right)\right)}=F\left(\overline{z\left(\ell_{k}\right)}\right)$ and $\overline{z\left(\ell_{k}\right)}=z\left(\ell_{-k}\right)$; and since the contour is symmetric, (4.9) becomes

$$
E_{t}=\frac{h}{\pi i} \sum_{k=N+1}^{\infty} e^{z\left(\ell_{k}\right) t} F\left(z\left(\ell_{k}\right)\right) z^{\prime}\left(\ell_{k}\right)
$$

Because of the exponential factor $e^{z\left(\ell_{k}\right) t}$, the terms in the sum decrease exponentially as $k \rightarrow \infty$ and in this case one commits only an exponentially small error whose contribution therefore can be neglected.

\subsubsection{Conditioning error}

To study the conditioning error in the application of the Laplace transform, recall that in (4.7), the approximation $-\tilde{f}(t)$ requires the evaluation of the transformed $F\left(z_{k}\right)=F\left(z\left(\ell_{k}\right)\right)$, for $k=-M ;-M+1 ; \ldots ; M-1 ; M$. In reality these evaluations are affected by round-off errors which means that the actual approximation that takes place is

$$
\tilde{F}\left(z_{k}\right)=F\left(z_{k}\right)+\rho_{k}, \quad k=-M,-M+1, \ldots, M-1, M,
$$

where $\rho_{k}>0$ is a small value such that $\left|\rho_{k}\right| \leqslant \epsilon$, with $\epsilon$ given by the machine precision. As a result (4.7) implies

$$
\check{f}_{M}(t)=\frac{h}{2 \pi i} \sum_{k=-M}^{M} e^{z_{k} t}\left(F\left(z_{k}\right)+\rho_{k}\right) z_{k}^{\prime}=\tilde{f}_{M}(t)+\frac{h}{2 \pi i} \sum_{k=-M}^{M} e^{z_{k} t} \rho_{k} z_{k}^{\prime} .
$$

We set

$$
f_{\rho}(t)=\frac{h}{2 \pi i} \sum_{k=-M}^{M} e^{z_{k} t} \rho_{k} z_{k}^{\prime}
$$

To see how the conditioning error affects the numerical results, we need to estimate kfqðtpk. Before we proceed, we state the following lemma [7]. Let 


$$
L(x)=1+\left|\ln \left(1-e^{-x}\right)\right|, \quad x>0 .
$$

The function $L$ is such that, $L(x) \rightarrow 1$ as $x \rightarrow \infty$ and $L(x) \sim|\ln x|$ as $x \rightarrow 0^{+}$.

Lemma 4.2. This function $L$ satisfies the following lemma

$$
\int_{0}^{\infty} e^{-\gamma \cosh x} d x \leqslant L(\gamma), \quad \gamma>0
$$

Proof. Consider the change of variable $\mathrm{m}=\cosh \mathrm{x}_{-} 1$ so that

$$
\int_{0}^{\infty} e^{-\gamma \cosh x} d x=e^{-\gamma} \int_{0}^{\infty} \frac{e^{-\gamma v}}{\sqrt{v(2+v)}} d v
$$

Set $a=\sinh ^{2}(1 / 2)$, so that

$$
\int_{0}^{a} \frac{d v}{\sqrt{v(2+v)}}=1, \quad \text { and } \quad \frac{1}{\sqrt{(k+a)(2+k+a)}} \leqslant \frac{1}{k+1}, \quad k \geqslant 0 .
$$

Thus

$$
e^{-\gamma} \int_{0}^{\infty} \frac{e^{-\gamma v}}{\sqrt{v(2+v)}} d v \leqslant e^{-\gamma}\left(1+\sum_{k=0}^{\infty} e^{-\gamma k} \int_{a+k}^{a+k+1} \frac{d v}{\sqrt{v(2+v)}}\right) \leqslant e^{-\gamma}\left(1+\sum_{k=0}^{\infty} \frac{e^{-\gamma k}}{k+1}\right)=e^{-\gamma}+L(\gamma)-1,
$$

which concludes the proof.

Now, we note that

$$
\begin{aligned}
\left\|f_{\rho}(t)\right\| & =\left\|\frac{h}{2 \pi i} \sum_{k=-M}^{M} e^{z_{k} t} \rho_{k} z_{k}^{\prime}\right\|=\left\|\frac{\tilde{\mu} e^{\tilde{\mu} t}}{2 \pi} h \sum_{k=-M}^{M} e^{\tilde{\mu} \sin \left(i \ell_{k}-\alpha\right) t} \rho_{k} \cos (i \ell-\alpha)\right\|, \quad \alpha \in(\pi / 4, \pi / 2) \\
& \leqslant \frac{\tilde{\mu} e^{\tilde{\mu} t}}{2 \pi} h \sum_{k=-M}^{M}\left\|e^{\tilde{\mu} \sin \left(i \ell_{k}-\alpha\right) t} \rho_{k} \cos (i \ell-\alpha)\right\| .
\end{aligned}
$$

The following two inequalities are proved in [7]. Here we re-state them in our context. One of them is 


$$
\left\|e^{\tilde{\mu} t \sin (i \ell-\alpha)} \rho_{k} \cos (i \ell-\alpha)\right\| \leqslant \rho e^{-\tilde{\mu} t \sin \alpha \cosh \ell} \cos (i \ell-\alpha),
$$

whereas the other one is

$$
e^{-\tilde{\mu} t \sin \alpha \cosh \ell} \cos (i \ell-\alpha) \leqslant \frac{1}{e \tilde{\mu} \varrho \sin \alpha t} e^{-(1-\varrho) \tilde{\mu} t \sin \alpha \cosh \ell}, \quad \varrho \in(0,1) .
$$

From (4.13) and (4.14), we deduce that

$$
\begin{aligned}
\left\|f_{\rho}(t)\right\| & \leqslant \frac{e^{\tilde{\mu} t} \rho}{2 \pi e \varrho \sin \alpha} \frac{h}{t} \sum_{k=-M}^{M} e^{-(1-\varrho) \tilde{\mu} t \sin \alpha \cosh \ell_{k}}, \\
& \leqslant \frac{e^{\tilde{\mu} t} \rho}{\pi e \varrho \sin \alpha} \frac{h}{t} \sum_{k=0}^{\infty} e^{-(1-\varrho) \tilde{\mu} t \sin \alpha \cosh \ell_{k}}, \\
& \leqslant \frac{e^{\tilde{\mu} t}}{\pi e \varrho \sin \alpha} \frac{\rho}{t} \int_{0}^{\infty} e^{-(1-\varrho) \tilde{\mu} t \sin \alpha \cosh \ell} .
\end{aligned}
$$

Using Lemma 4.2, we obtain

$$
\left\|f_{\rho}(t)\right\| \leqslant \frac{e^{\tilde{\mu} t}}{\pi e \varrho \sin \alpha} \frac{\rho}{t}[L((1-\varrho) \tilde{\mu} t \sin \alpha)] .
$$

We note that (4.16) is independent of $F$ and propagates moderately with respect to $\tilde{\mu} t$.

In summary (4.10), the argument mentioned just after (4.12) and (4.16) imply that the total error is fully controllable as long as we choose the optimal values of the associated parameters. The derivation of these parameters is described below.

\subsection{Derivation of the optimal contour parameters}

In this subsection, we present the derivation of the contour parameters. The basic idea is taken from [17]. In that paper, the authors derived the following convergence estimate for a family of hyperbolic contours (4.5):

$$
E_{d}=\mathcal{O}\left(e^{-2 \pi(\pi / 2-\alpha) / h}\right), \quad E_{-d}=\mathcal{O}\left(e^{\tilde{\mu} t-2 \pi \alpha / h}\right), \quad h \rightarrow 0 .
$$

Therefore, the total discretization error on the strip $(-d, d)$, denoted by $E_{r}$, is given by

$$
E_{r}=E_{d}+E_{-d} \text {. }
$$

Moreover, the truncation error that we found satisfies

$$
E_{t}=\mathcal{O}\left(e^{\tilde{\mu} t(1-\sin \alpha \cosh (h M))}\right), \quad M \rightarrow \infty .
$$

To estimate the optimal parameters of the contour, an asymptotic balance of the three errors, i.e., $\mathrm{E}_{\mathrm{t}} ; \mathrm{E}_{\mathrm{d}} ; \mathrm{E} \_\mathrm{d}_{\mathrm{d}}$ is required. To this end, we set 


$$
E_{d}=E_{-d}=E_{t}
$$

so that

$$
\frac{-2\left(\frac{\pi}{2}-\alpha\right)}{h}=\tilde{\mu} t-\frac{2 \pi \alpha}{h}=\tilde{\mu} t(1-\sin \alpha \cosh (h M)) .
$$

We solve these equations for $h, \tilde{\mu}$ and $\alpha \in(\pi / 4, \pi / 2)$. From the first equation in (4.20), we deduce that

$$
\tilde{\mu} t=\pi \frac{4 \alpha-\pi}{h},
$$

and

$$
\cosh (h M)=\frac{2 \alpha}{(4 \alpha-\pi) \sin \alpha}
$$

Denoting $h M$ by $A(\alpha)$, we obtain from (4.22)

$$
A(\alpha)=h M=\cosh ^{-1}\left(\frac{2 \alpha}{(4 \alpha-\pi) \sin \alpha}\right) .
$$

Finally, (4.21) and (4.23) give

$$
\tilde{\mu}=\frac{4 \pi \alpha-\pi^{2}}{A(\alpha)} \frac{M}{t}, \quad \text { and } \quad h=\frac{A(\alpha)}{M} .
$$

The corresponding convergence estimate can now be derived from $h$ and $\tilde{\mu}$ in (4.24) as

$$
E_{M}=\mathcal{O}\left(e^{-B(\alpha) M}\right), \quad \text { where } \quad B(\alpha)=\frac{\pi^{2}-2 \pi \alpha}{A(\alpha)}, \quad M \rightarrow \infty .
$$

To derive the optimal contours parameters, note that as $B(\alpha)$ increases, $E M$ decreases exponentially. Thus $E M$ attains its optimum value at the maximum of $B(\alpha)$ over the interval (л/4; л/2). A numerical computation of $B(\alpha)$ shows that the maximum attained at

$$
\alpha_{\star}=1.1721
$$

With the optimal value of $\alpha$ given by $\alpha_{\star}$ above, we can now compute optimal value of $\tilde{\mu}$ and $h$ from (4.24) and express them as

$$
h_{\star}=\frac{1.0818}{M}, \quad \text { and } \quad \tilde{\mu}_{\star}=4.4921 \frac{M}{t} .
$$

The optimal contour is therefore given by

$$
z=4.4921 \frac{M}{t}(1+\sin (i \ell-1.1721)), \quad \ell \in \mathbb{R},
$$

and the optimal error as

$$
E_{M}^{\star}=\mathcal{O}\left(e^{-2.32 M}\right)=\mathcal{O}\left(10.2^{-M}\right), \quad M \rightarrow \infty .
$$

From the above, observed that the optimal error while using the Laplace transform will be exponentially small. In the next subsection, we discuss its practical implementation to solve the problem under consideration.

\subsection{Application of the Laplace transform to the jump-diffusion model} In the Laplace domain, Eq. (3.24) becomes 


$$
z \widehat{V}-V_{0}=(B+\lambda H) \widehat{V}+\frac{\epsilon_{1}(s)}{z}-\frac{\epsilon_{2}(s)}{z+r},
$$

where $\widehat{V}$ is the unknown solution in this domain. The boundary conditions are given as

$$
\left.\begin{array}{ll}
\widehat{V}(0, z) & =0, \\
\widehat{V}\left(S_{\max }, z\right) & =\frac{K e^{\max }}{z}-\frac{K}{z+r}
\end{array}\right\} .
$$

From (4.30), we deduce

$$
[z I-(B+\lambda H)] \widehat{V}=V_{0}+\frac{\epsilon_{1}(s)}{z}-\frac{\epsilon_{2}(s)}{z+r} .
$$

Application of the inversion formula (4.6) yields

$$
V(t)=\frac{h}{2 \pi i} \int_{-\infty}^{\infty} e^{z(\ell) t} \widehat{V} z^{\prime}(\ell) d \ell .
$$

Using the trapezoidal rule (4.7) truncated at $M$ and the symmetry of the contour (4.5), we obtain the value of the options as

$$
V_{M}(t)=\operatorname{Re}\left\{\frac{h}{\pi} \sum_{k=0}^{M} e^{\circ} e^{z_{k}} t \widehat{V}_{k} z_{k}^{\prime}\right\},
$$

where ${ }^{\circ}$ indicates that the first term is divided by 2 and the vector $\widehat{V}_{k}$ is solved for each $k$ as

$$
\widehat{V}_{k}=\left[z_{k} I-(B+\lambda H)\right]^{-1}\left(V_{0}+\frac{\epsilon_{1}(s)}{z_{k}}-\frac{\epsilon_{2}(s)}{z_{k}+r}\right), \quad k=0, \ldots, M .
$$

In next section, we present some numerical results illustrating the proposed method.

\section{Numerical results}

In this section we illustrate the performance of our Laplace inversion method (ILT) and the spectral domain decomposition method (SDDM) in solving the European jumpdiffusion model. We also evaluate the $\Delta$ and $\Gamma$ of this option.

To apply the SDDM, we split the domain into two sub-domains consisting of equal number of grid points, with the domain boundary placed at $o$ where the initial condition is non-smooth. For numerical illustration of our approach, we consider the European jump-diffusion model with the following parameters $K=10 ; \sigma=0.2 ; \lambda=0.1$; $y=0.3 ; \mu=0 ; r=0.02 ; T=0.25$ and truncated domain $s \min =-3$ and $s \max =1.5$.

For numerical comparison, we consider the computational time and the maximum absolute errors which is calculated using the formula

$$
\max _{t \in[0, T]}\left|V(t)-V_{N}(t)\right|,
$$

where $V(t)$ is the analytical solution and $V_{N}(t)$ is the numerical solution at time $t$.

Though the number of contour points may vary with the problem at hand, for the European option, we carry out different simulations by keeping the number of grid 
points $N$ fixed and varying the number of points on the contour. For different value of $N$, we found that the optimal number of contour grid points varies from 20 to 30 .

Table 1 displays the numerical results obtained by using the Crank-Nicholson $(\mathrm{CN})$ and the ILT method for time integration and the Finite difference method (FDM) for spatial discretization. The numerical results obtained by using $\mathrm{CN}$ and ILT methods for time integration and SDDM for spatial discretization are presented in Table 2. The computations are performed for the same set of parameters except that different grids are used for comparison. The ILT method is clearly more accurate than the $\mathrm{CN}$ for both the finite difference and spectral domain decomposition discretizations. For example, for 180 grid points we get an order of accuracy of $10^{-3}$ in 0.144 seconds and 150 time step using $\mathrm{CN}$, whereas we obtain an order of accuracy of $10^{-4}$ in 0.061 seconds and 20 contour points using the ILT method in Table 1.

Table 1

Numerical results obtained by using Crank-Nicholson and inversion Laplace transform method for time and standard finite difference method for the spatial (asset) discretization with $\mathrm{M}$ number of points on the contour and using the parameters $K=10 ; \sigma=0.2 ; \lambda=0.1 ; \gamma=0.3 ; \mu=0 ; r=0.02 ; T=0.25$; $x_{\min }=-2, x_{\max }=1.5$.

\begin{tabular}{|c|c|c|c|c|c|c|c|}
\hline \multicolumn{4}{|c|}{$\mathrm{CN}$ and FDM } & \multicolumn{4}{|c|}{ ILT and FDM } \\
\hline$N$ & Time steps & Error & Time (s) & $N$ & $M$ & Error & Time (s) \\
\hline 40 & 50 & $1.40 \mathrm{E}-2$ & 0.013 & 40 & 20 & $1.04 \mathrm{E}-2$ & 0.012 \\
\hline 90 & 60 & $4.60 \mathrm{E}-3$ & 0.027 & 90 & 20 & $2.80 \mathrm{E}-3$ & 0.022 \\
\hline 180 & 150 & $1.50 \mathrm{E}-3$ & 0.144 & 180 & 20 & $6.86 \mathrm{E}-4$ & 0.061 \\
\hline 650 & 340 & $3.14 \mathrm{E}-4$ & 5.240 & 650 & 20 & $1.25 \mathrm{E}-5$ & 1.083 \\
\hline 900 & 650 & $3.60 E-4$ & 22.355 & 900 & 20 & $2.66 \mathrm{E}-5$ & 2.473 \\
\hline
\end{tabular}

Table 2

Numerical results obtained by using Crank-Nicholson and inversion Laplace Transform methods for time and spectral domain decomposition method for the spatial (asset) discretization with $\mathrm{M}$ number of points on the contour and the parameters $K=10 ; \sigma=0.2 ; \lambda=0.1 ; \gamma=0.3 ; \mu=0 ; r=0.02 ; T=0.25$; $x_{\min }=-2, x_{\max }=1.5$.

\begin{tabular}{|c|c|c|c|c|c|c|c|}
\hline \multicolumn{4}{|c|}{$\mathrm{CN}$ and SDDM } & \multicolumn{4}{|c|}{ ILT and SDDM } \\
\hline$N$ & Time steps & Error & Time (s) & $N$ & M & Error & Time (s) \\
\hline 5 & 200 & $2.490 \mathrm{E}-1$ & 0.049 & 5 & 20 & $6.20 \mathrm{E}-1$ & 0.047 \\
\hline 10 & 200 & $6.300 \mathrm{E}-2$ & 0.050 & 20 & 20 & $1.30 \mathrm{E}-3$ & 0.054 \\
\hline 20 & 300 & $1.300 \mathrm{E}-3$ & 0.076 & 20 & 20 & $1.30 \mathrm{E}-3$ & 0.048 \\
\hline 35 & 380 & $1.310 \mathrm{E}-4$ & 0.120 & 35 & 20 & $1.31 \mathrm{E}-5$ & 0.062 \\
\hline 50 & 400 & $1.240 \mathrm{E}-4$ & 0.177 & 50 & 20 & $2.68 \mathrm{E}-8$ & 0.071 \\
\hline 70 & 650 & $7.650 \mathrm{E}-5$ & 0.315 & 70 & 20 & $3.19 \mathrm{E}-10$ & 0.084 \\
\hline
\end{tabular}

Table 2 displays improved results compared to those from Table 1 for both ILT and CN methods as the number of spatial points is significantly fewer for the SDDM method to get the same level of accuracy than FDM. This illustrates the superiority of the SDDM over FDM.

Tables 1 and 2 also show the computational time taken by both approaches. We see that the ILT and SDDM methods are faster than the CN and FDM methods respectively. Furthermore, from Table 2 we note that despite computing bloc matrices in the SDDM as opposed to the computation of sparse matrices in the FDM discretization, the advantage of the SDDM is that the number of grid points $N$ is usually smaller to achieve higher order at a faster speed. 
In Fig. 2(a) we have plotted the numerical and exact solution of the European jumpdiffusion problem. To illustrate the effect of the jump, we also plotted the solution of the Black-Scholes equation without jump. Fig. 2(b) shows the graphical accuracy of the numerical solution for $N=50$ in each sub-domains.

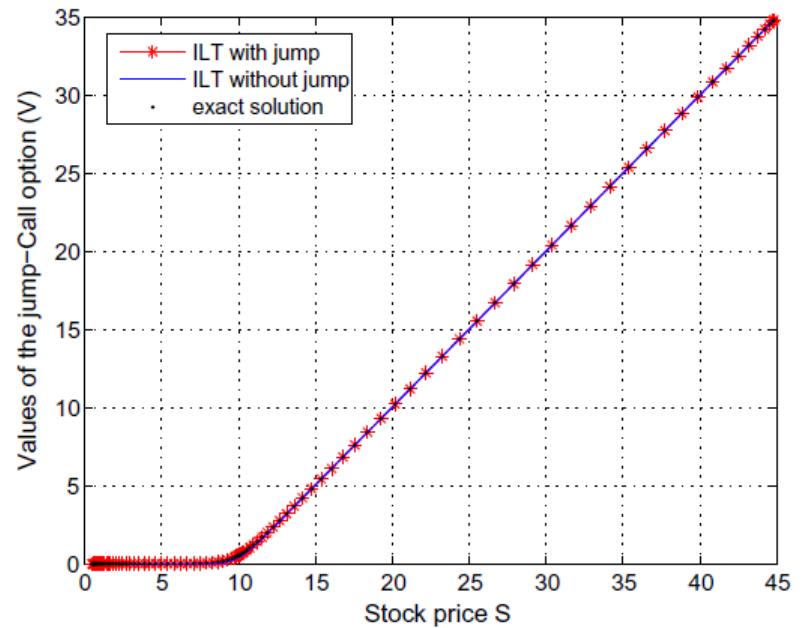

(a)

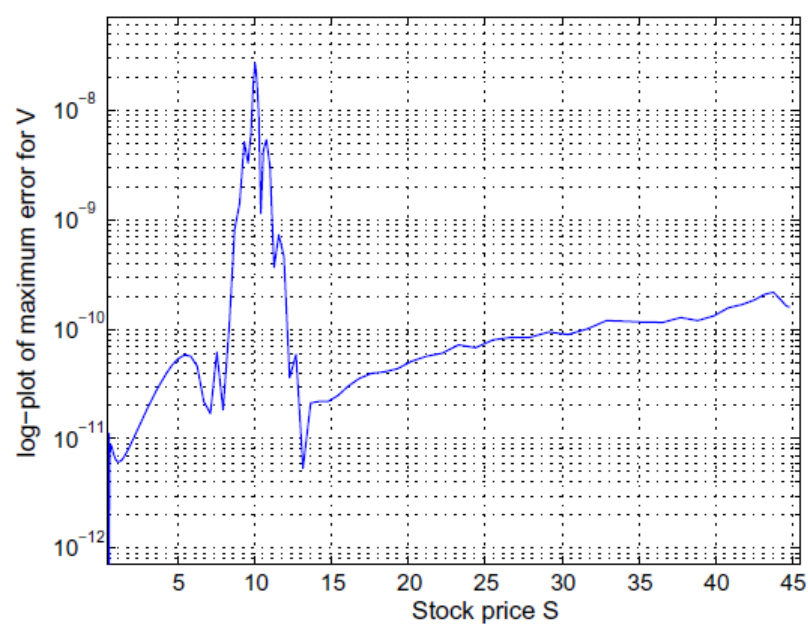

(b)

Fig. 2. (a) Inverse Laplace transform (ILT) solution of the European call option with jump, without jump and the exact solution; (b) maximum error occurred during the evaluation of the European call option with jump using inverse Laplace transform (ILT). The domain was divided into two sub-domains. Computing parameters were taken as $K=10 ; \sigma=0.2 ; r=0.02 ; \gamma=0.2 ; \lambda=0.5, \mu=0 ; T=0.25 ; x_{\max }=1.5, x_{\min }=-3 ; N=50$ in each sub-domain.

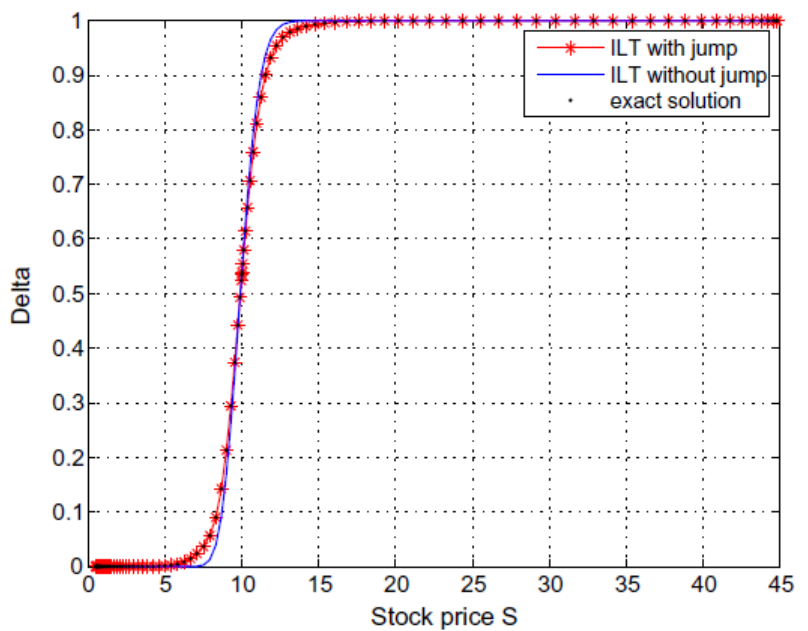

(a)

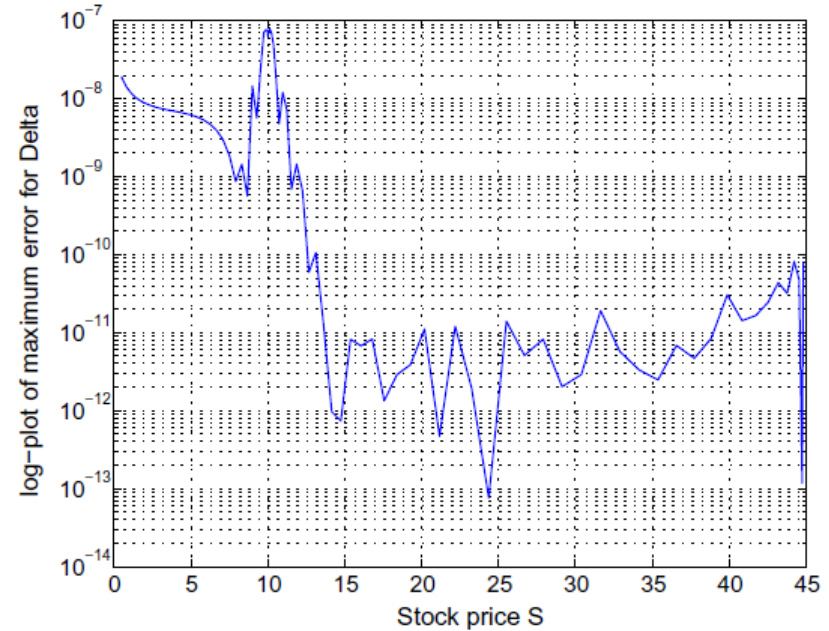

(b)

Fig. 3. (a) Inverse Laplace transform (ILT) approximation of the $\Delta$ with jump, without jump and the exact solution; (b) maximum errors occurred during the computation of $\Delta$. Computing parameters were taken as $K=10 ; \sigma=0.2 ; r=0.02 ; \gamma=0.2 ; \lambda=0.5, \mu=0 ; T=0.25 ; x_{\max }=1.5, x_{\min }=-3 ; N=50$ in each subdomain. 


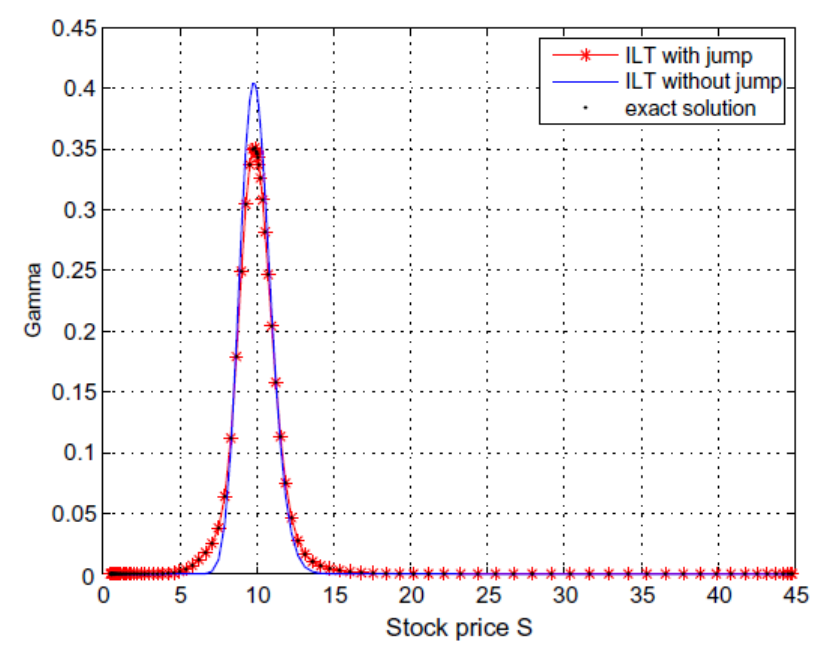

(a)

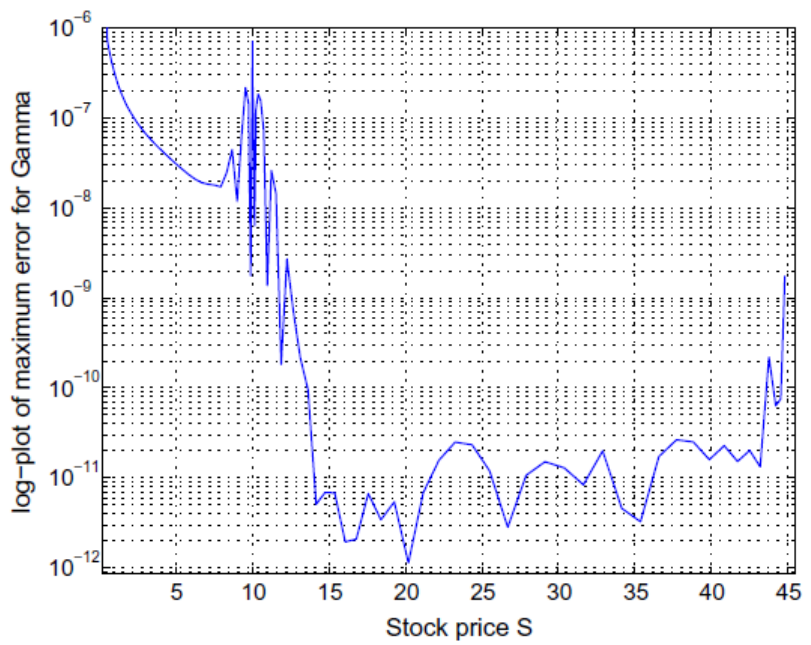

(b)

Fig. 4. (a) Inverse Laplace transform (ILT) approximation of the $\Gamma$ with jump, without jump and the exact solution; (b) maximum errorS occurred during the computation of $\Gamma$. Computing parameters were taken as $K=10 ; \sigma=0.2 ; r=0.02 ; \gamma=0.2 ; \lambda=0.5, \mu=0 ; T=0.25 ; x_{\max }=1.5, x_{\min }=-3 ; N=50$ in each subdomain.

Figs. 3(a) and 4(a) show the numerical values of $\mathrm{D}$ and $\mathrm{C}$ respectively with and without jump. We also plot exact values of these Greeks. The convergence error observed in Figs. 3(b) and 4(b) demonstrates exponential accuracy of the proposed method.

\section{Concluding remarks and scope for future research}

We have presented a new approach to price European options with jump. Our approach consisted of the inverse Laplace transform (ILT) method as a time integrator and the spectral domain decomposition method (SDDM) as a spatial discretization method.

The SDDM uses piecewise high order linear rational interpolants represented pointwise on a Chebyshev grid points in each sub-domain. The choice of the decomposition method was motivated by the need to obtain high accurate results since a direct application of spectral method (without decomposition) is not well suited to the non-smooth initial condition of the PIDE. To approximate the convolution integral that represents the jump process, we used the same grid points as for the differential part.

We compared both the ILT and SDD methods with the Crank-Nicholson method and finite difference discretizations. The proposed approach is exponentially convergent in space and time, with the further advantage that it computes the solution at a particular time level directly and thus no time stepping is required. Spectral accuracy is observed not only in the evaluation of the option but also in the valuation of the Greeks D and C. Moreover, the computational time taken by this method is significantly less as compared to the one taken by $\mathrm{CN}$ and FDM.

The numerical results presented in this paper suggest that the spectral domain decomposition method is very robust for pricing financial derivatives. 
We are currently investigating the extensions of this approach to more complicated model such as American jump-diffusion model. Preconditioning of the block matrix in SDDM to further speed up the computation may also be investigated.

\section{Acknowledgment}

The authors acknowledge the anonyms referees for their valuable comments and suggestions which helped the presentation of the work. The two authors E. Ngounda and E. Pindza acknowledge to the Agence National des Bourses du Gabon for the financial support. K.C. Patidar's research was supported by the South African National Research Foundation. 


\section{References}

[1] Abate J, Valko PP. Multi-precision Laplace transform inversion. Int J Numer Meth Eng 2004;60(5):979-93.

[2] Andersen L, Andreasen J. Jump-diffusion processes: volatility smile fitting and numerical methods for option pricing. Rev Derivat Res 2000;4:231-62. [3] Berrut JP, Mittelmann HD. Rational interpolation through the optimal attachment of poles to the interpolating polynomial. Numer Algor 2000;23(4):315-28.

[4] Davies B, Martin B. Numerical inversion of Laplace transform: a survey and comparison of methods. J Comput Phys 1979;33(1):1-32.

[5] Duffy DG. On the numerical inversion of Laplace transform: a comparison of the three new methods on characteristic problems from applications. ACM Trans Math Software 1993;19(3):333-59.

[6] Duffy DG. Transform methods for solving partial differential equations. 2nd ed. Boca Raton, FL: Chapman \& Hall/CRC; 2004.

[7] López-Fernández M, Palencia C. On the numerical inversion of the Laplace transform of certain holomorphic mappings. Appl Numer Math 2004;51(23):289-303.

[8] Martensen VE. Zur numerischen Auswertung uneigentlicher Integrale. Z Angew Math Mech 1968;48:T83-5. [9] Merton RC. Option pricing when underlying stock returns are discontinous. J Financ Econom 1976;3:125-44.

[10] Narayanan GV, Beskos DE. Numerical operational methods for timedependent linear problems. Int J Numer Meth Eng 1982;18(12):1829-54. [11] Schneider C, Werner W. Some new aspect of rational interpolation. Math Comput 1986;47(175):285-99.

[12] Spiegel MR. Theory and problems of laplace transforms. New York: Schaum Publishing Co.; 1965.

[13] Talbot A. The accurate numerical inversion of Laplace transforms. IMA J Appl Math 1979;23(1):97-120.

[14] Tangman DY, Gopaul A, Bhuruth M. Exponential time integration and Chebychev discretisation schemes for fast pricing of options. Appl Numer Math 2008;58(9):1309-19.

[15] Tee W, Trefethen LN. A rational collocation with adaptively transformed Chebyshev grid points. SIAM J Sci Comput 2006;28(5):1798-811. [16] Trefethen LN. Is Gauss quadrature better than Clenshaw-Curtis? SIAM Rev 2008;50(1):67-87.

[17] Weideman JAC, Trefethen LN. Parabolic and hyperbolic contours for computing the Bromwich integral. Math Comput 2007;76(259):1341-56.

[18] Zhu W, Kopriva D. A Spectral element method to price European options.I. Single asset with and without jump-diffusion. J Sci Comput 2009;39(2):222-43.

[19] Zvan R, Forsyth PA, Vetzal KR. Penalty methods for American option with stochastic volatility. J Comput Appl Math 1998;91(2):199-218. 